\title{
Relation of VEGF to the risk, severity and prognosis of preeclampsia
}

\author{
Original Emam Awad ${ }^{1}$ and Mahmoud Abulgasem El-Hamedi ${ }^{2}$ \\ Article \\ ${ }^{1}$ Department of Obstetrics and Gynecology Faculty of Medicine Tanta, Egypt \\ ${ }^{2}$ Department of Obstetrics and Gynecology Faculty of Medicine Zawia - Libya
}

\begin{abstract}
Objective: The aim of this work was to find out if serum level of vascular endothelial growth factor shows changes with the onset of preeclampsia that could be used as predictor for preeclampsia.

Patients and Methods: This study included 60 primigravidae women divided into three groups, group (I) included 20 normal women, group (II) included 20 women with mild preeclampsia and group (III) included 20 women with severe preeclampsia.VEGF was measured in all groups and compared

Results: VEGF concentrations measured in severe preeclampsia group were significantly higher than mild preeclampsia group and control group. Thus, VEGF concentrations could be used as a predictor for diagnosis and follow up of severity of preeclampsia. VEGF had significant value for discrimination between patients with PE (mild and severe) and controls with cutoff value $>632.4 \mathrm{ng} / \mathrm{l}$ which had a sensitivity of $(82.5 \%)$ and specificity of $(85 \%)$. Also, serum VEGF concentration for discrimination between patients with severe PE and those with mild or no PE was $>1335.5 \mathrm{ng} / \mathrm{l}$ with specificity of $(92.5 \%)$ and sensitivity of (90\%). VEGF had significant value for prediction of adverse fetal outcomes with cutoff value $>741.5 \mathrm{ng} / \mathrm{l}$ which had a sensitivity of $(95.65 \%)$ and specificity of $(72.97 \%)$.

Conclusion: According to the previous data obtained, serum VEGF level can be used as a predictor for preeclampsia.
\end{abstract}

Key Words: VEGF, preeclampsia, pregnancy

Received: 15 November 2019, Accepted: 21 April 2019

Corresponding Author: Mahmoud Abulgasem El-Hamedi, MD, Obstetrics and Gynecology Department, Faculty of Medicine Zawia, Libya, Tel.: +2 01142831371, E-mail: kaainih@yahoo.com

ISSN: 2090-7265, August 2019, Vol. 9, No. 3

\section{INTRODUCTION}

In preeclampsia, there is an immunologically mediated deficiency in the trophoblastic invasion of the placental bed by spiral arterioles resulting in poorly perfused fetoplacental unit, which in turn secrets mediators into the maternal blood causing the multisystem pathological changes which are characteristic for preeclampsia. VEGF possesses many of the characteristics required for a candidate circulating factor to be one of these preeclamptic mediators ${ }^{[1]}$.

Vascular endothelial growth factor (VEGF) is a glycosylated glycoprotein which has a vasodilator and vascular permeability enhancing effects, in addition to angiogenic and a potent endothelial cell mitogenic property ${ }^{[2]}$.

Preeclampsia (PE) is a clinical syndrome present in 5 to $8 \%$ of all pregnant women and remains as a major cause of maternal and neonatal mortality and morbidity worldwide ${ }^{[3]}$.

It is characterized by endothelial dysfunction, hypertension and proteinuria after $20 \mathrm{w}$ of gestation and/or a fetal syndrome ${ }^{[4]}$.

In preeclampsia, the remodeling of spiral uterine arteries is disturbed, probably as a result of the insufficient cytotrophoblast invasion in the maternal uterus, which causes hypoxia. As a result of hypoxia, the placenta releases placental factors with pro- or anti-angiogenic action such as vascular endothelial growth factor (VEGF) and soluble VEGF receptor-1 (sVEGFR-1 $)^{[5]}$.

On the other hand, preeclampsia is characterized by an angiogenic imbalance at both placental and systemic level, which causes endothelial dysfunction and the clinical manifestation of this disease ${ }^{[6]}$.

Preeclampsia also remains a leading cause of prematurity as placenta delivery is currently the only way to successfully treat the disorder. The etiology and pathogenesis of this pregnancy-specific syndrome are unclear. Accumulated evidence suggests that PE may be the result of an imbalance in angiogenic factors ${ }^{[4]}$.

Recent studies have hypothesized that vascular endothelial growth factor (VEGF) and its receptors are implicated in the pathophysiology of preeclampsia ${ }^{[7]}$.

In normal pregnancy, there is a remodeling of uterine spiral arteries through the invasion of cytotrophoblast, which facilitates blood circulation and the transport of oxygen and nutrient substances to the fetus. The balance between pro-angiogenic and anti-angiogenic factors modulates this vascular remodeling, angiogenesis being an important process in implantation and placentation ${ }^{[8]}$. 
Vascular endothelial growth factor (VEGF) and placental growth factor (PGF) are potent regulators of vascular development during embryogenesis, as well as blood vessel formation and survival in adulthood ${ }^{[9]}$.

Another factor involved in the neovascularization, soluble form tyrosine kinase like receptor type-1 (sFlt-1), is an anti-angiogenic circulating splice variant of VEGFR-1. It acts by adhering to the receptor- binding domain of the angiogenic factors, preventing their normal interaction with their cell surface receptor and leading to endothelial dysfunction ${ }^{[10]}$.

In stage 1 of early-onset preeclampsia, impaired extra villous trophoblast invasion into maternal spiral arteries causes poor vascular remodeling and induces placental and endothelial damage. In stage 2 , these damaged tissues release antiangiogenic factors such as the soluble form tyrosine kinase like receptor type-1 (sFlt-1) and soluble endoglin (sENG), a co-receptor for transforming growth factor (TGF)-1 and -3 , which induces maternal intravascular systemic inflammatory responses and endothelial dysfunction, resulting in hypertension and proteinuria after 20 weeks' gestation, especially in earlyonset preeclampsia ${ }^{[11]}$.

Endocrine gland vascular endothelial growth factor (EG-VEGF) is the major prokineticin in the female reproductive tract. In the placenta, EG-VEGF and its receptors are highly expressed ${ }^{[12]}$.

The syncytiotrophoblast layer (ST) is the main layer where EG- VEGF is mainly localized in with a mild expression in the cytotrophoblast layer $(\mathrm{CT})^{[13]}$.

Endocrine gland vascular endothelial growth factor (EG-VEGF) receptor PROKR1 is abundant in the CT and the placental microvascular endothelial cells, whereas PROKR2 is expressed by ST and extra villous trophoblasts $(\mathrm{EVT})^{[14]}$.

Endocrine gland vascular endothelial growth factor (EG-VEGF) and its receptors show a dynamic profile throughout pregnancy. In the placenta, EG-VEGF, PROKR1 and PROKR2 are predominantly expressed during the first trimester of pregnancy. During early pregnancy, EG-VEGF/PROKR1 peaks at 8-11 weeks of gestation and then gradually decreases by the end of the first trimester, whereas PROKR2 expression is maintained over the first trimester. In non- pregnant women, circulating EG-VEGF levels are around $50 \mathrm{pg} / \mathrm{mL}$. During pregnancy, these levels significantly increase fivefold during the first trimester $(250 \mathrm{pg} / \mathrm{mL})$ and then gradually decrease to reach those observed in non-pregnant women by the end of the second trimester of pregnancy ${ }^{[1]}$.

\section{AIM OF THE WORK}

To measure serum level of vascular endothelial growth factor (VEGF) in patients with pre-eclampsia and correlate it with severity of the disease to be used as predictor for pre-eclampsia.

\section{PATIENTS AND METHODS}

This study was conducted at Tanta University Hospital in the inpatient wards and outpatient clinic of Obstetrics and Gynecology Department in the period from Jan 2016 till June 2016 on 60 patients.

\section{Patients were divided into 3 groups:}

Group (I): (N=20) including normal pregnant women with blood pressure $<140 / 90$ and no proteinuria. (Control Group).

Group (II): $(\mathrm{N}=20)$ including patients with mild preeclampsia.

Group (III): ( $\mathrm{N}=20)$ including patients with severe preeclampsia.

All recruited patients gave a written informed consent about the study and the procedures done in this study were:

\section{Inclusion criteria:}

1. Age: $(20-35 \mathrm{Y})$.

2. Gestational age: after 20 weeks.

3. Singleton pregnancy.

4. Primigravida.

\section{Exclusion criteria:}

1. Medical disorders with pregnancy such as chronic hypertension, chronic renal disease and diabetes mellitus.

2. Congenital fetal malformations (CFM).

3. Intra uterine demise (IUD).

4. Other concurrent obstetric disorders such as placenta previa, rupture membranes and vaginal bleeding.

5. Smoking and alcohol intake.

6. Connective tissue diseases like SLE.

\section{METHODS}

\section{All cases in the groups were subjected to:}

1. Detailed history (personal, present, past, menstrual and family).

2. Examination (general and local):

C. General examination: head and neck, chest, abdomen (fundal grip for gestational age, polyhydramnios, oligohydramnios and auscultation of fetal heart), pelvis, upper and lower limbs for edema.

D. Local examination: P.V for pelvic assessment, vulva for edema or varicose veins and premium for any abnormalities.

3. Investigations: Laboratory including: urine analysis, complete blood count (CBC), serum blood glucose, kidney functions, liver function and vascular 
endothelial growth factor (VEGF) was measured in serum by Enzyme Linked Immunosorbent Assay (ELISA).

\section{Abdominal ultrasound:}

- Fetal viability.

- Fetal biometry (Biparietal diameter, abdominal circumference and Femur length)

- Fetal activity.

- Exclude fetal anomalies.

- Amniotic fluid volume.

- Placental grade and location.

- Mild pre-eclampsia was diagnosed in women whose blood pressure (140-159/90-109) and /or proteinuria $\leq 0.3 \mathrm{~g} / 24 \mathrm{hrs}$ by dipstick test.

- Severe pre-eclampsia was diagnosed in women whose blood pressure $\geq 160 / 110$ and/or proteinuria $\geq 5 \mathrm{~g} / 24 \mathrm{hrs}$ by dipstick test or with either of the following:

A. Symptoms of severity like (headache, blurring of vision, epigastric pain, right hypogastric pain, ultrasound evidence of IUGR, nausea and vomiting).

B. Signs and/or symptoms of end organ affection (oliguria, thrombocytopenia and impaired liver function).

- $\quad$ Pitting edema in preeclampsia was noticed in lower limbs, vulva, abdomen and face or through rapid weight gain during antenatal care. According to degree of preeclampsia, edema would be mild or severe.

Vascular endothelial growth factor (VEGF) ELISA testing

\section{Test principle:}

The kit uses a double-antibody sandwich enzymelinked immunosorbent assay (ELISA) to assay the level of human vascular endothelial growth factor (VEGF) in samples.

\section{RESULTS}

This study was conducted in Tanta University Hospital on 60 pregnant women whom VEGF was compared with baseline blood pressure, proteinuria, edema and adverse outcome either maternal or fetal.

\section{Patients were divided into 3 groups:}

Group (I): (N=20) including normal pregnant women with blood pressure $<140 / 90$ and no proteinuria (Control Group).

Group (II): $(\mathrm{N}=20)$ including patients with mild preeclampsia.

Group (III): ( $\mathrm{N}=20)$ including patients with severe preeclampsia.

Data were analyzed and the results of this study were summarized in the following tables and figures:

The results of this study showed that there is a positive correlation between vascular endothelial growth factor (VEGF) and severity of preeclampsia and this will be discussed later.

Table 1: Clinical characteristics of the three study groups

\begin{tabular}{|c|c|c|c|c|c|c|c|c|}
\hline \multirow{2}{*}{ Variable } & \multicolumn{2}{|c|}{ Control $(n=20)$} & \multicolumn{2}{|c|}{ Mild PE $(n=20)$} & \multicolumn{2}{|c|}{ Severe PE $(n=20)$} & \multirow{2}{*}{$\mathrm{F}(\mathrm{df}=2 \& 57)$} & \multirow{2}{*}{$P$-value } \\
\hline & Mean & $\mathrm{SD}$ & Mean & SD & Mean & SD & & \\
\hline Age (years) & 27.1 & 5.1 & 26.4 & 4.5 & 27.9 & 5.0 & .479 & 0.622 \\
\hline Gestational age (weeks) & 33.3 & 4.2 & 32.1 & 5.0 & 34.2 & 3.6 & 1.146 & 0.325 \\
\hline $\mathrm{SBP}(\mathrm{mmHg})$ & 119 & 10 & $147 \dagger$ & 5 & $181 \dagger t$ & 16 & 155.770 & $*<0.001$ \\
\hline DBP $(\mathrm{mmHg})$ & 76 & 6 & $96 \dagger$ & 5 & $112 \dagger t$ & 3 & 280.040 & $*<0.001$ \\
\hline MAP (mmHg) & 90 & 7 & $113 \dagger$ & 4 & $135 \dagger t$ & 6 & 324.335 & $*<0.001$ \\
\hline \multicolumn{9}{|l|}{ Proteinuria } \\
\hline Nil & 20 & $100.0 \%$ & 0 & $0.0 \%$ & 0 & $0.0 \%$ & 55.934 & $*<0.001$ \\
\hline $1+$ & 0 & $0.0 \%$ & 8 & $40.0 \%$ & 0 & $0.0 \%$ & & \\
\hline $2+$ & 0 & $0.0 \%$ & 12 & $60.0 \%$ & 0 & $0.0 \%$ & & \\
\hline $3+$ & 0 & $0.0 \%$ & 0 & $0.0 \%$ & 20 & $100.0 \%$ & & \\
\hline Edema & & & & & & & 56.149 & $*<0.001$ \\
\hline Nil & 20 & $100.0 \%$ & 0 & $0.0 \%$ & 0 & $0.0 \%$ & & \\
\hline Mild & 0 & $0.0 \%$ & 20 & $100.0 \%$ & 2 & $10.0 \%$ & & \\
\hline Severe & 0 & $0.0 \%$ & 0 & $0.0 \%$ & 18 & $90.0 \%$ & & \\
\hline
\end{tabular}

* F,F statistic; df, degree of freedom. 
Table 2: Incidence of adverse Mather and fetal outcomes in the three study groups.

\begin{tabular}{|c|c|c|c|c|c|c|c|}
\hline \multirow{2}{*}{ Variable } & \multicolumn{2}{|c|}{ Control $(\mathrm{n}=2)$} & \multicolumn{2}{|c|}{ Mild PE $(n=20)$} & \multicolumn{2}{|c|}{ Severe PE $(n=20)$} & \multirow{2}{*}{$P$-value } \\
\hline & $\mathrm{n}$ & $\%$ & $\mathrm{n}$ & $\%$ & $\mathrm{~N}$ & $\%$ & \\
\hline Accidental hemorrhage & 0 & $0.0 \%$ & 0 & $0.0 \%$ & 3 & $15.0 \%$ & 0.100 \\
\hline Pulmonary edema & 0 & $0.0 \%$ & 0 & $0.0 \%$ & 2 & $10.0 \%$ & 0.322 \\
\hline HELLP syndrome & 0 & $0.0 \%$ & 0 & $0.0 \%$ & 4 & $20.0 \%$ & $<0.030$ \\
\hline Eclampsia & 0 & $0.0 \%$ & 0 & $0.0 \%$ & 3 & $15.0 \%$ & 0.100 \\
\hline Impaired kidney function & 0 & $0.0 \%$ & 2 & $10.0 \%$ & 3 & $15.0 \%$ & 0.353 \\
\hline Need for termination & 0 & $0.0 \%$ & 0 & $0.0 \%$ & 1 & $5.0 \%$ & 1.000 \\
\hline preterm labor & 1 & $5.0 \%$ & 7 & $35.0 \%$ & 10 & $50.0 \%$ & $*<0.005$ \\
\hline IUGR & 0 & $0.0 \%$ & 2 & $10.0 \%$ & 2 & $10.0 \%$ & 1.000 \\
\hline Prematurity & 1 & $5.0 \%$ & 7 & $35.0 \%$ & 10 & $50.0 \%$ & $*<0.005$ \\
\hline IUFD as a complication of PE & 0 & $0.0 \%$ & 1 & $5.0 \%$ & 3 & $15.0 \%$ & 0.310 \\
\hline Fetal distress & 0 & $0.0 \%$ & 2 & $5.0 \%$ & 4 & $20.0 \%$ & 0.344 \\
\hline Overall incidence of adverse fetal outcomes & 1 & $5.0 \%$ & 12 & $60.0 \%$ & 19 & $95.0 \%$ & $*<0.002$ \\
\hline Number of cases with adverse fetal outcomes & 1 & $5.0 \%$ & 8 & $40 \%$ & 14 & $70 \%$ & $*<0.005$ \\
\hline
\end{tabular}

Table 3: Comparison of VEGF level in the three study groups.

\begin{tabular}{|c|c|c|c|c|c|c|c|c|}
\hline \multirow{2}{*}{ Variable } & \multicolumn{2}{|c|}{ Control $(n=20)$} & \multicolumn{2}{|c|}{ Mild PE $(n=20)$} & \multicolumn{2}{|c|}{ Severe PE $(n=20)$} & \multirow{2}{*}{$\mathrm{F}(\mathrm{df}=2 \& 57)$} & \multirow{2}{*}{ P-value } \\
\hline & Mean & $\mathrm{SD}$ & Mean & $\mathrm{SD}$ & Mean & SD & & \\
\hline VEGF (ng/l) & 357.0 & 161.2 & $863.2 \dagger$ & 314.4 & $2204.4 \div \S$ & 728.2 & 83.468 & $*<0.001$ \\
\hline
\end{tabular}

Table 4: Relation between VEGF level and number of cases with or without adverse fetal outcomes

\begin{tabular}{lccccccc}
\hline \multirow{2}{*}{ Variable } & \multicolumn{2}{c}{ adverse neonatal outcomes $(\mathrm{n}=37)$} & \multicolumn{2}{c}{ Adverse neonatal outcomes (n=23) } & Mann- Whitney U & \multirow{2}{*}{ Z } \\
\cline { 2 - 5 } & Median & Interquartile range & Median & Interquartile range & & \\
\hline VEGF (ng/l) & 527.9 & 298.0 to 778.4 & 1734.1 & 1041.7 to 2518.5 & 97.0 & 4.995 \\
\hline
\end{tabular}

\section{DISCUSSION}

Preeclampsia is associated with excessive production of a soluble form of the vascular endothelial growth factor receptor-1 (VEGF-1), termed soluble form tyrosine kinase like receptor type-1 (sFlt-1). This placental- derived factor is believed to be a key contributor to the pathogenesis of $\mathrm{PE}^{[15]}$.

Most investigators believed that vascular endothelial growth factor receptor-1 might not be a receptor transmitting a mitogenic signal, but rather receptor able to inhibit the activity of VEGF on vascular endothelium by preventing binding of VEGF to VEGFR-2. ${ }^{[16]}$

The vascular endothelial growth factor (VEGF) is a glycosylated glycoprotein selectively mitogenic for endothelial cells and appears to play a major role in the mediation of angiogenesis. VEGF also has a vasodilator effect in addition to its ability of increasing the microvascular permeability ${ }^{[16]}$.

Despite intense research efforts, the pathogenesis of preeclampsia is still mysterious, but it is mostly multifactorial[ ${ }^{[17]}$.

The pathophysiology of preeclampsia likely involves maternal, fetal and placental factors. Abnormalities in the development of placental vasculature early in pregnancy may result in relative placental under-perfusion /hypoxia/ ischemia which then leads to release of antiangiogenic factors into maternal circulation that alter maternal systemic endothelial function and cause hypertension and other manifestations of the disease $\mathrm{e}^{[18]}$.

In this study, vascular endothelial growth factor (VEGF) was higher in the two study groups (mild and severe preeclampsia) compared to those in the control group and was higher in the group of severe preeclampsia than in the group of mild preeclampsia with significant difference between the three study groups ( $P$ value $<0.001)$.

In this study, mean VEGF was $2204.4 \mathrm{ng} / 1$ in severe preeclampsia group versus $863.2 \mathrm{ng} / 1$ in mild preeclampsia group and $357 \mathrm{ng} / \mathrm{l}$ in the control group ( $P$ value $<0.001)$. Thus, serum free VEGF is potentially useful as a marker in prediction of severity of preeclampsia.

VEGF level was significantly higher in their cases. The optimal cutoff point for VEGF was calculated as 600.5 $\mathrm{ng} / \mathrm{L}$ to differentiate the cases and the controls with $64 \%$ sensitivity and $100 \%$ specificity and $(P$ value $<0.001)$. There was a significant increase in median serum level of VEGF in severe cases compared to the mild cases. The optimal cutoff 
point for VEGF was calculated as $673.5 \mathrm{ng} / \mathrm{L}$ to differentiate mild and severe cases, with $93.3 \%$ sensitivity and $90 \%$ specificity and $(P \text { value }<0.001)^{[17]}$.

T Celik et al. 2013 study demonstrated that maternal serum VEGF level in this preeclampsia group was higher than the control group ; it was $314.45 \pm 260.74 \mathrm{pg} / \mathrm{ml}$ in preeclampsia group versus $14.2 \pm 14.54 \mathrm{pg} / \mathrm{ml}$ in the control group; the difference was statistically significant ${ }^{20]}$. Celik et al. 2013 explained the increase of VEGF in the preeclampsia group by endothelial cell damage that occurs in preeclampsia increases VEGF production during endothelial repair ${ }^{[21]}$

Increased concentration of circulating VEGF may cause extravasation of plasma proteins and proteinuria, which is the main symptom of preeclampsia ${ }^{[22]}$. The other possibility is that VEGF is secreted from the placenta and its production is increased in response to local ischemia in the placental infarction areas as a result VEGF increases in response to placental hypoxia ${ }^{[23]}$.

Hunter et al. 2000 study demonstrated that evaluated median serum VEGF level in their preeclampsia group was $51.7 \mathrm{ng} / \mathrm{ml}$, about 4 times the median level in the control group which was $13.9 \mathrm{ng} / \mathrm{ml}$. The women with the two highest VEGF levels in the preeclampsia group had thrombocytopenia, with platelet levels of 100,000 $\mathrm{mL}$. Additionally, Hunter's study also demonstrated that concentrations of VEGF were significantly elevated in preeclampsia cases for several weeks before the clinical manifestation of the disease. The concentrations of VEGF that occurred before the clinical disease manifests may also be indicative of the timing of the onset with the highest levels at 20 and 30 weeks occurring in those who developed PE early ${ }^{[24]}$.

Masoura's study concluded that in preeclampsia an angiogenic imbalance between VEGF and sFlt-1 exists and plays an important role in pathogenesis of endothelial dysfunction $^{[26]}$.

In this study, the mean of the gestational age at delivery was shorter in women of the two study groups (mild and severe preeclampsia), in severe preeclampsia was 34.2 weeks versus 36.1 weeks in mild preeclampsia and 38.3 weeks in controls with significant difference between the three study groups ( $P$ value $<0.001)$. This is agreeing with Masoura et al. 2014 in their study that showed that the gestational week at delivery and birth weight was significantly shorter in the preeclampsia group than controls.

Kurtoglu et al. 2016 study explained that the gestational week at delivery of the same 3 groups which was shorter in women of the two study groups in severe preeclampsia was 34.5 weeks versus 36 weeks in mild preeclampsia and 38.4 weeks in controls. Also, Maged et al. 2016 study showed that the pregnancy duration at delivery in severe preeclampsia group was significantly shorter than that in mild preeclampsia and control groups, so this is agreeing with our results ${ }^{[19,25,26]}$.
In this study, the incidence of HELLP syndrome was high in severe preeclampsia group, while it was absent in mild preeclampsia group and the control group with significant difference between the three study groups ${ }^{[19,25,26]}$ $(P$ value $<0.030)$. Also, the need for preterm termination of pregnancy was higher in women of the two study groups (mild and severe preeclampsia) compared to those in the control group and in the group of severe preeclampsia was higher than in the group of mild preeclampsia with significant difference between the three study groups (P value $<0.001$ ). Also, other adverse obstetric outcomes were reported in the severe preeclampsia group like pulmonary edema, eclampsia and impaired kidney function.

This is in agreement with Maged et al. 2016 study that showed significant difference between women with severe preeclampsia and women in the other two groups (mild and control) in terms of maternal complications such as HELLP syndrome and eclampsia. Also, this is in agreement with Singh et al. 2016 study that showed that HELLP syndrome was the most common maternal complication seen in patients with preeclampsia, whereas pulmonary edema was seen in $3.6 \%$ of patients and maternal mortality rate was $1.8 \%[26,27]$.

Similar results have also been obtained by Murphy and Stirrat 2000 study that showed that $21 \%$ of cases had developed HELLP syndrome, $13 \%$ had renal failure and $1.4 \%$ had eclampsia. In another study conducted by Minire et al. $2013,3.2 \%$ of patients had eclampsia, $4.2 \%$ had HELLP syndrome and $5.5 \%$ had pulmonary edema which is in agreement with our study ${ }^{[28,29]}$.

In this study, the incidence of prematurity and overall adverse fetal outcomes were higher in women of the two study groups (mild and severe preeclampsia) compared to those in the control group with significant difference between the three study groups $(P$ value $<0.005)$ for prematurity and $(P$ value $<0.002)$ for overall adverse fetal outcomes.

This is in agreement with Maged et al. 2016 study that showed, regarding neonatal complications, that the two study groups (mild and severe preeclampsia) had significantly lower Apgar scores than the control group and admission to the Neonatal Intensive Care Unit (NICU) was more than in the preeclampsia groups. Moreover, severe preeclampsia was associated with perinatal death. Also, similar results were obtained by Masoura et al. 2014 in their case control study that showed a higher rate of neonates with prematurity, IUGR and NICU admissions was observed in the preeclampsia group compared to the control group which is in agreement with our results ${ }^{[26,30]}$.

The analysis that was performed between the biomarkers assayed and the neonatal outcomes in the preeclampsia group revealed significant correlation between late preterm, early term and VLBW and sFlt-1. On the other hand, a trend was observed between lower serum concentrations of the angiogenic marker VEGF and prematurity, as well as NICU admission, so this doesn't agree with our result that showed that median 
(interquartile range) serum level of VEGF was higher in the group with adverse neonatal outcomes than in the group with no adverse neonatal outcomes with significant difference $(P$ value $<0.00)$. From Masoura et al. 2014 study, Maynard et al. 2003 explained the pathophysiology of preeclampsia by defective placentation results in ischemic placenta which initiates the release of sFLt-1 that binds to angiogenic factor VEGF, blocking its action of placental neovascularization and depriving the maternal blood vessels from its vasodilator effect in addition inducing systemic endothelial dysfunction, which results in clinical manifestations of preeclampsia and its associated adverse maternal and neonatal outcomes ${ }^{[30,31]}$.

Receiver operating characteristics (ROC) curve analysis of VEFG as a marker used for discrimination between the two case groups (mild and severe preeclampsia) and the control group showed that area under curve (AUC) equal to 0.967 with cutoff value $>632.4 \mathrm{ng} / \mathrm{L}$ which revealed sensitivity of $82.5 \%$ and specificity of $85 \%$. ( $P$ value $<0.001)$.

Receiver operating characteristics (ROC) curve analysis of VEFG as a marker used for discrimination between the severe preeclampsia group and the mild preeclampsia group showed that area under curve (AUC) equal to 0.980 with cutoff value $>1355.5 \mathrm{ng} / \mathrm{L}$ which revealed sensitivity of $90 \%$ and specificity of $85 \%$. (P value $<0.001)$.

Receiver operating characteristics (ROC) curve analysis of VEFG as a marker used for prediction of adverse fetal outcomes in the studied groups showed that area under curve (AUC) equal to 0.886 with cutoff value $>741.5 \mathrm{ng} / \mathrm{L}$ which revealed sensitivity of $95.65 \%$ and specificity of 72.97\%. (P value $<0.001)$.

\section{CONCLUSION AND RECOMMENDATION}

In conclusion, the current study indicates that increased serum vascular endothelial growth factor (VEGF) level can be used as a predictor of transformation of pregnancy from normal to preeclampsia at a cutoff value of VEGF $>632.4$ $\mathrm{ng} / \mathrm{l}$ and from mild to severe preeclampsia at a cutoff value of VEGF $>1335.5 \mathrm{ng} / 1$. Also, adverse pregnancy outcomes can be predicted at a cutoff value of VEGF $>741.5 \mathrm{ng} / 1$.

Vascular endothelial growth factor (VEGF) level is recommended to be used as a marker to predict transformation of the disease and recognition of adverse pregnancy outcomes.

Future studies should focus on VEGFR-1 and its role in pathogenesis of preeclampsia.

\section{CONFLICTS OF INTEREST}

There are no conflicts of interest.

\section{REFERENCES}

1. Lyall, Stephenc, Robson , and Judith NB. (2013) spiral artery remodeling and trophoblast invasion in preeclampsia and Feral growth restriction relationship to clinical outcome, "hypertension 62 , No.6 $1046-54$.
2. Shibuya and Masabumi (2013): Vascular endotelial growth Factor and its receptor system : physiological functions in angiogenesis and pathological roles in various diseases. Journal of biochemistry 153 , no 1-13-19.

3. Steeger E, von Dadelszen $\mathrm{p}$ and Duvekot J. Preeclampsia. Lancet 2010:376; 631-44.

4. Chedraui P, Solis E and Bocci. Fete-placental nitric oxide, asymmetric dimethylarginine and vascular endothelial growth factor (VEGF) levels and VEGF gene polymorphisms $\mathrm{m}$ severe preeclampsia. J Matern Fetal Neonatal Med 2013:26; 226-32.

5. Lee E, Oh M and Jung J. The levels of circulating vascular endothelial growth factor and soluble Flt$1 \mathrm{~m}$ pregnancies complicated by preeclampsia. J Korean Med Sci 2007: 22;94-8.

6. Lucia M, Gabriela C, Gabriela Z, and Florin S. Maternal/newborn VEGF-C936T interaction and its influence on the risk severity and prognosis of preeclampsia, as well as on the maternal angiogenic profile J Matern Fetal Neonatal Med, 2014: $2 ; 1754-60$.

7. Molvarec A, Szarka A and Walentin S. circulating angiogenic factors determined by electrochemiluminescence immunoassay in relation to the clinical features and laboratory parameters in women with pre-eclampsia. Hypertens Res 2010: $33 ; 892-8$.

8. Szentpe'teri I, Rab A and Kornya L. Gene expressiOn patterns of vascular endothelial growth factor (VEGF-A) in human placenta from pregnancies with intrauterine growth restriction. J Matern Fetal Neonatal Med 2013: 26; 984-9.

9. Rodrigues P, Furriol J and Tormo E. The singlenucleotide polymorphisms +936 CIT VEGF and 710 CIT VEGFR 1 are associated with breast cancer protection $\mathrm{m}$ a Spanish population. Breast Cancer Res Treat 2012: 133; 769-78.

10. Ribatti D. The discovery of the placental growth factor and its role in angiogenesis: a historical review. Angiogenesis 2008: 11; 215-21.

11. Gina A. Smith, Gareth W. Fearnley, Michael A. Harrison, Darren C, Omlinson T, Stephen B. Wheatcroft and Sreenivasan P.Vascular endothelial growth factors: multitasking functionality in metabolism, health and disease, 2015: 38; 753-63.

12. Reimer T, Rohrmann H and Stubert J. Angiogenic factors and acute-phase proteins in serum samples of preeclampsia and HELLP patients: a matchedpair analysis. J Matern Fetal Neonatal Med 2013: 26; 263-9.

13. Evans J, Catalano Rand Morgan K, "Prokineticin 1 signaling and gene regulation in early human pregnancy," Endocrinology, 2008: 149; 2877-87. 
14. Hoffann P, Feige $J$ and Alfaidy N. "Expression and oxygen regulation. of endocrine gland-derived vascular endothelial growth factor/prokineticin-1 and its receptors in human placenta during early pregnancy," Endocrinology, 2006: 147; 1675-84.

15. Hoffann P, Saoudi $Y$ and Benharouga M. "Role ofEG-VEGF in human placentation: physiological and pathological implications," Journal of Cellular and Molecular Medicine, 2009: 13; 2224-35.

16. Zhou Q, Liu H and Qiao F (2010): VEGF deficit is involved in endothelium dysfunction in preeclampsia. Huazhong Univ Sci Technolog Med Sci. 30(3):370-4.

17. Takahashi S (2011): Vascular endothelial growth factor (VEGF), VEGF receptors and their inhibitors for angiogenic tumour therapy. Biol. Pharm. Bull; vol 34(12):1785-88.

18. Roberts JM, Pearson GD, Cutler JA and Lindheimer MD (2003): Summary of the NHLBI working group on research on hypertension during pregnancy "Hypertension in pregnancy; vol. 22, no.11:109-27.

19. Brosens I, Pijnenborg R, Vercruysse L and Romero R (2011): "The great Obstetrical Syndromes" are associated with disorders of deep placentation. Am J Obstet Gynecol; 204:193.

20. Kurtoglu E, Avci B, Kokcu A, Celik H, Dura MC, Malatyalioglu E, and Ozdemir AZ (2016): J Matern Fetal Neonatal Med; 29(12): 1987-92.

21. Celik H, Avaci B and Isik Y (2013): Vacular endothelial growth factor and endothelin-1 levels in normal pregnant women and pregnant women with preeclampsia. J Obstet Gynecol; 33:355-58

22. Tsurumi Y, Murohara T, Krasinsk K, Chen D, Witzenbichler B and Kearney M (1997). Reciprocal relation between VEGF and NO in the regulation of endothelial integrity. Nature Medicine 3: 879-86.
23. Ferrara N, Houck K, Jakeman J, Leung DW (1992). Molecular and biological properties of the vascular endothelial growth factor family of proteins. Endocrine Reviews; 13: 18.

24. Levine RJ, Maynard SE, Qian C, Lim KH, England LJ and Yu KF (2004): Circulating angiogenic factors and the risk of preeclampsia. New England Journal of Medicine; 350: $672-83$.

25. Hunter A, Aitkenhead M, Caldwell C, McCracken G, Wilson D and McClure N (2000): Hypertension; 36:965-9.

26. Masoura S, Kalogiannidis I, Makedou K, Theodoridis T, Koiou K, Gerou S, Athanasiadis A and Agorastos T (2014): European Journal of Obstetrics and Gynecology and Reproductive Biology 175, 119-23.

27. Maged AM, Aid G, Bassiouny N, Eldin SD, Dahab S and Ghamry KN (2016): Association of biochemical markers with the severity of preeclampsia. Int $\mathrm{J}$ Obstet Gynecol; vol.136, no. (2):138-44.

28. Singh A, Chawla S, Pandey D, Jahan N and Anwar A (2016): Fetomaternal outcome in cases of preeclampsia in a tertiary care referral hospital in Delhi, India: A retrospective analysis. Int J Sci Study; vol.4, no. (2):100-03.

29. Murphy DJ and Stirrat GM (2000): Mortality and morbidity associated with early-onset preeclampsia. Hypertens Pregnancy; 19:221-31.

30. Minire A, Mirton M, Imri V, Lauren M and Aferdita M (2013): Maternal complications of preeclampsia. Med Arch; 67:339-41.

31. Maynard SE, Man JY, Merchan J, Lim KH, Li J, Mondal S, Libermann TA, Morgan JP, Sellke FW and Stillman IE (2003): Excess placental soluble Fms-like tyrosine kinase 1 (sFlT-1) may contribute to endothelial dysfunction, hypertension and proteinuria in preeclampsia. J Clin Invest 111, 600-02. 